\title{
Solution of the Time Dependent Schrödinger Equation and the Advection Equation via Quantum Walk with Variable Parameters
}

\author{
Shinji Hamada, Masayuki Kawahata, Hideo Sekino \\ Toyohashi University of Technology, Toyohashi, Japan \\ Email: hamada@adsim.tut.ac.jp, sekino@tut.jp
}

Received June 10, 2013; revised July 10, 2013; accepted August 1, 2013

Copyright (C) 2013 Shinji Hamada et al. This is an open access article distributed under the Creative Commons Attribution License, which permits unrestricted use, distribution, and reproduction in any medium, provided the original work is properly cited.

\begin{abstract}
We propose a solution method of Time Dependent Schrödinger Equation (TDSE) and the advection equation by quantum walk/quantum cellular automaton with spatially or temporally variable parameters. Using numerical method, we establish the quantitative relation between the quantum walk with the space dependent parameters and the "Time Dependent Schrödinger Equation with a space dependent imaginary diffusion coefficient" or "the advection equation with space dependent velocity fields". Using the 4-point-averaging manipulation in the solution of advection equation by quantum walk, we find that only one component can be extracted out of two components of left-moving and right-moving solutions. In general it is not so easy to solve an advection equation without numerical diffusion, but this method provides perfectly diffusion free solution by virtue of its unitarity. Moreover our findings provide a clue to find more general space dependent formalisms such as solution method of TDSE with space dependent resolution by quantum walk.
\end{abstract}

Keywords: Quantum Walk; Quantum Cellular Automaton; Time Dependent Schrödinger Equation; Advection Equation

\section{Introduction}

Quantum walk is a mechanical system evolved by a discrete local unitary transformation and is regarded as a quantum version of the classical random walk $[1,2]$. In recent years, relations between the quantum walk and continuous quantum wave equations were shown [3-9] and more recently a certain view point was added to the relation between the quantum walk and the Time Dependent Schrödinger Equation (TDSE) [10].

There are some formalisms on the broadly-defined quantum walk. Here we use the formalism usually called as quantum cellular automaton (QCA). Namely, we simply consider it as a mechanical system evolved by a unitary transformation by a banded matrix.

A quantum walk can be most easily conceived by comparing it with classical random walk. One-dimensional classical random walk is defined by a transition probability matrix $\hat{P}_{i j}$ applying a probability distribution $\rho_{i}$.

$$
\rho_{i}(t+\Delta t)=\sum_{j} \hat{P}_{i j} \rho_{j}(t)
$$

The conservation of probability requires

$$
\sum_{i} \hat{P}_{i j}=1
$$

And the requirement that the transition occurs only between neighboring grid points means that $\hat{P}_{i j}$ should be a banded matrix.

In quantum walk, where probability amplitude evolves instead, this transition probability matrix is replaced by the unitary banded matrix. Namely,

$$
\Psi_{i}(t+\Delta t)=\sum_{j} \hat{U}_{i j} \Psi_{j}(t), \sum_{k} \hat{U}_{k i}^{*} \hat{U}_{k j}=\delta_{i j}
$$

Incidentally the difference between a random process and a quantum process is that in the latter case the probability is given by squaring the amplitude. In order to conserve total probability, the transformation must be unitary,

$$
\sum_{i}\left|\hat{U}_{i j}\right|^{2}=1
$$

and therefore formally the correspondence relation 
$\hat{P}_{i j} \approx\left|\hat{U}_{i j}\right|^{2}$ holds.

While it is apparently easy to make some transition probability matrix that satisfies the conservation of probability (Equation (1.2)), it requires some devices to construct a unitary banded matrix.

However we note that the unitary banded matrix represented on discrete space is quite similar to the twoscale transformation matrix in an orthogonal wavelet with compact support [11].

One of the easiest way to construct the unitary banded matrix is to use the product of trivial unitary banded matrices as shown in Figure 1. Here we refer to a block diagonal matrix whose block diagonal components are 2 $\times 2$ unitary matrices as a trivial unitary banded matrix.

By using the product of trivial unitary matrices in the RHS, we can construct a non-trivial unitary banded matrix in the LHS of Figure 1. In fact, reversely it is true that any translationally invariant unitary banded matrix can be factorized in the form of the RHS (see Appendix B).

In general we can introduce space dependent parameters $(\theta(x), \phi(x), b(x))$ in the quantum walk (see Appendix A).

$$
U(x)=\left(\begin{array}{cc}
\cos \theta(x) & i \sin \theta(x) e^{-i \phi(x)} \\
i \sin \theta(x) e^{i \phi(x)} & \cos \theta(x)
\end{array}\right) e^{-i b(x)}
$$

Here we use $U$ in place of $\mathrm{E}$ and $\mathrm{F}$ in Figure 1.

In these parameters, $b(x)$ means potential term in TDSE (see Appendix A).

$\phi(x)$ is a local gauge transformation parameter (namely redefinition of the phase of wave function), but we don't discuss space dependent $\phi(x)$ here while it is an interesting subject.

\section{Time Dependent Schrödinger Equation with Space Dependent Imaginary Diffusion Coefficient}

Here we discuss space dependent parameters $(\theta(x), b(x))$ in the TDSE-type quantum walk.

$$
U(x)=\left(\begin{array}{cc}
\cos \theta(x) & i \sin \theta(x) \\
i \sin \theta(x) & \cos \theta(x)
\end{array}\right) e^{-i b(x)}
$$

By the relation $1 / m=\tan \theta$ (see Appendix A), $\theta(x)$ can be interpreted as a parameter for the space dependent

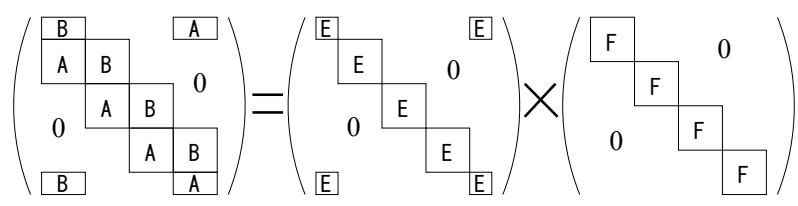

Figure 1. Product of trivial unitary banded matrices $(E, F$ are $2 \times 2$ unitary matrices). imaginary diffusion coefficient $(1 /(2 m))$.

The Hamiltonian that emerges in the continuum limit evolution equation

$$
i \frac{\partial \Psi(x, t)}{\partial t}=H \Psi(x, t)
$$

may have the following form and/or their linear combinations because $H$ must be Hermitian and

$$
\begin{aligned}
H=-\frac{1}{2 m} D^{2} \text { for constant } A(x) . & \\
H_{\alpha}=- & \frac{1}{2} A(x)^{\alpha} D A(x)^{2-2 \alpha} D A(x)^{\alpha} \\
= & -\frac{1}{2}\left(A(x)^{2} D^{2}+2 A(x) A(x)_{x} D\right. \\
& \left.+\alpha A(x) A(x)_{x x}+\alpha(1-\alpha) A(x)_{x}^{2}\right)
\end{aligned}
$$

where $A(x)=1 / \sqrt{m(x)}, A(x)_{x}, A(x)_{x x}$ are first and second derivatives with respects to space $x$ and $D \equiv \frac{\partial}{\partial x}$.

We show some examples as follows.

$$
\begin{aligned}
H_{0} & =-\frac{1}{2} D A(x) A(x) D, \\
H_{1} & =-\frac{1}{2} A(x) D D A(x), \\
H_{\frac{1}{2}} & =-\frac{1}{2} \sqrt{A(x)} D A(x) D \sqrt{A(x)} \\
& =-\frac{1}{2}(\sqrt{A(x)} D \sqrt{A(x)})^{2}
\end{aligned}
$$

By making $H_{\frac{1}{2}}$ as a basis for which theoretical solution can be obtained easily, $H_{\alpha}$ can be rewritten as

$$
\begin{aligned}
H_{\alpha}= & H_{\frac{1}{2}} \\
& -\frac{1}{2}\left(\left(\alpha-\frac{1}{2}\right) A(x) A(x)_{x x}-\left(\alpha-\frac{1}{2}\right)^{2} A(x)_{x}^{2}\right)
\end{aligned}
$$

We must note that additional potential term emerges when $\alpha$ is changed.

For the case of $\mathrm{H}$ as the linear combination of $H_{\alpha}$, we have more general form

$$
H=H_{\frac{1}{2}}-\left(\alpha^{\prime} A(x) A(x)_{x x}+\beta A(x)_{x}^{2}\right)
$$

Since analytical derivationof $\alpha^{\prime}, \beta$ is not straightforward because of the broken translational invariance, we determine $\alpha^{\prime}, \beta$ using a numerical method.

Namely, the solution for

$H+\left(\alpha^{\prime} A(x) A(x)_{x x}+\beta A(x)_{x}^{2}\right)$ is calculated using TDSEtype quantum walk with space dependent $\theta(x)$ with 
additional potential term $\left(\alpha^{\prime} A(x) A(x)_{x x}+\beta A(x)_{x}^{2}\right)$ and compared with the analytical solution for $H_{1}$. $\frac{1}{2}$

We determine $\alpha^{\prime}, \beta$ so that the two solutions completely coincide.

The used $2 \times 2$ matrix of quantum walk is

$$
\begin{aligned}
& U(x)=\left(\begin{array}{cc}
\cos \theta(x) & i \sin \theta(x) \\
i \sin \theta(x) & \cos \theta(x)
\end{array}\right) e^{-i b(x)} \\
& \text { where } \tan \theta(x)=A(x)^{2}, \\
& b(x)=\theta(x)+\alpha^{\prime} A(x) A(x)_{x x}+\beta A(x)_{x}^{2}
\end{aligned}
$$

(Note that for the space dependent $\theta(x)$, phase compensation term $\theta(x)$, the first term in $b(x)$ is crucial and leads to meaningless results without it).

In our numerical method, we use periodic boundary condition for the range [0,1], and use two types of $A(x)$.

(More precisely, in the actual calculation the range $[0,1]$ was mapped to the range $[0,512])$

1) sine function type

$$
A(x)=\frac{A_{0}}{1+\epsilon \sin (2 \pi x)}\left(A_{0}=0.5, \epsilon=0.5\right)
$$

2) elliptic function type

$$
\begin{gathered}
A(x)=\frac{2 \pi}{4 K(k)} \frac{A_{0}}{d n(4 K(k) x, k)} \\
\left(A_{0}=0.5, k^{2}=0.9\right)
\end{gathered}
$$

where $4 K(k)$ is the period along the real axis of Jacobi's elliptic function

Both type of $A(x)$ satisfies

$$
\int_{0}^{1} \frac{\mathrm{d} x}{A(x)}=\frac{1}{A_{0}}
$$

In order to calculate the theoretical solution for

$$
\begin{aligned}
i \frac{\partial \psi(t, x)}{\partial t} & =H_{\frac{1}{2}} \psi(t, x) \\
& =-\frac{1}{2}(\sqrt{A(x)} D \sqrt{A(x)})^{2} \psi(t, x)
\end{aligned}
$$

we can simply reduce it to the solution of the free fields TDSE $\psi_{0}(t, x)$.

$$
\begin{aligned}
& i \frac{\partial \psi_{0}(t, x)}{\partial t}=-\frac{1}{2} D^{2} \psi_{0}(t, x) \\
& \psi(t, x)=\frac{\psi_{0}\left(t A_{0}^{2}, B(x)\right)}{\sqrt{A(x)}} \\
& \text { where } B(x) \equiv \int_{0}^{x} \frac{A_{0}}{A\left(x^{\prime}\right)} \mathrm{d} x^{\prime}
\end{aligned}
$$

(Note that as $B(x+1)=B(x)+1$, the periodicity $\psi(t, x+1)=\psi(t, x)$ is guaranteed $)$.

Concrete function forms for the $B(x)$ are

$$
\begin{aligned}
& B(x)=x-\frac{\epsilon}{2 \pi}(\cos (2 \pi x)-1) \\
& B(x)=\frac{1}{2 \pi} \arg (\operatorname{cn}(4 K(k) x, k)+i s n(4 K(k) x, k))
\end{aligned}
$$

for sine function type and elliptic function type respectively.

We use the fact that theoretical solution of the free field TDSE for the Gaussian wave packet can be written [12] as

$$
\begin{aligned}
& \psi_{00}(t, x)=\sqrt{\frac{C(t)}{C(0)}} \exp \left(C(t)(x-B)^{2}\right) \\
& \text { where } \frac{1}{C(t)}=\frac{1}{C(0)}+2 i t
\end{aligned}
$$

and we use periodic superposition of this $\left(\psi_{0}(t, x)=\sum_{n} \psi_{00}(t, x-n)\right)$ for the periodic boundary condition.

Here $\mathrm{C}, \mathrm{B}$ are complex numbers and $\mathrm{B}$ is constant in general (Note that as $\mathrm{C}, \mathrm{B}$ are complex numbers, the wave packet center of $|\Psi(t, x)|^{2}$ is not B but changes with time).

We used $\psi_{0}(t=0, x)=\exp \left(-(10(x-0.5))^{2}\right)$ for the initial wave packet.

We show the result of the numerical solution in Figures 2-5.

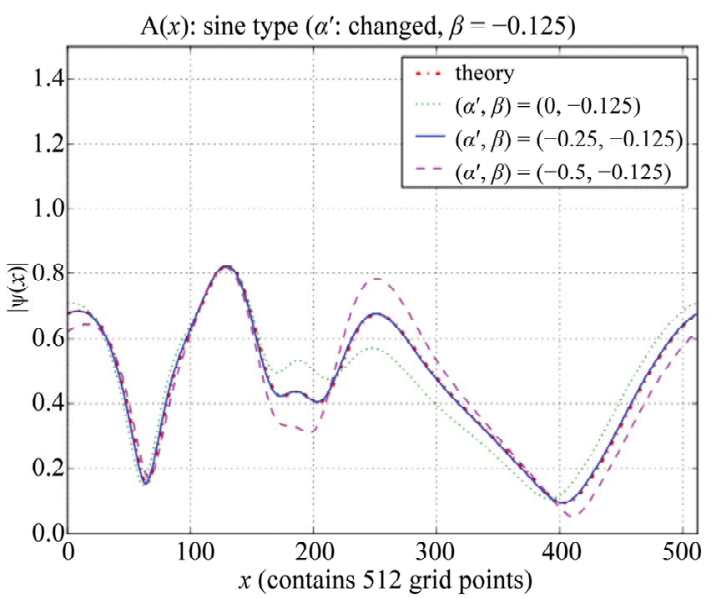

Figure 2. Parameter fitting for sine function type A(x) (after 50000 walks) dash-dotted red: theoretical solution, dotted green: $\left(\alpha^{\prime}, \beta\right)=(0,-0.125)$, solid blue: $\left(\alpha^{\prime}, \beta\right)=(-0.25$, $-0.125)$, dashed magenta: $\left(\alpha^{\prime}, \beta\right)=(-0.5,-0.125)$. Here, $\beta=$ -0.125 is fixed and $\alpha^{\prime}$ is varied. At $\alpha^{\prime}=-0.25$, the quantum walk solution coincides with the theoretical solution. 512 grid points are used. Absolute values of two-point-averaged $\psi$ are plotted. $\left(\psi_{\text {ave } 2}(t, x)=(1 / 2)(\psi(t, x)+\psi(t, x+\Delta x))\right)$. 


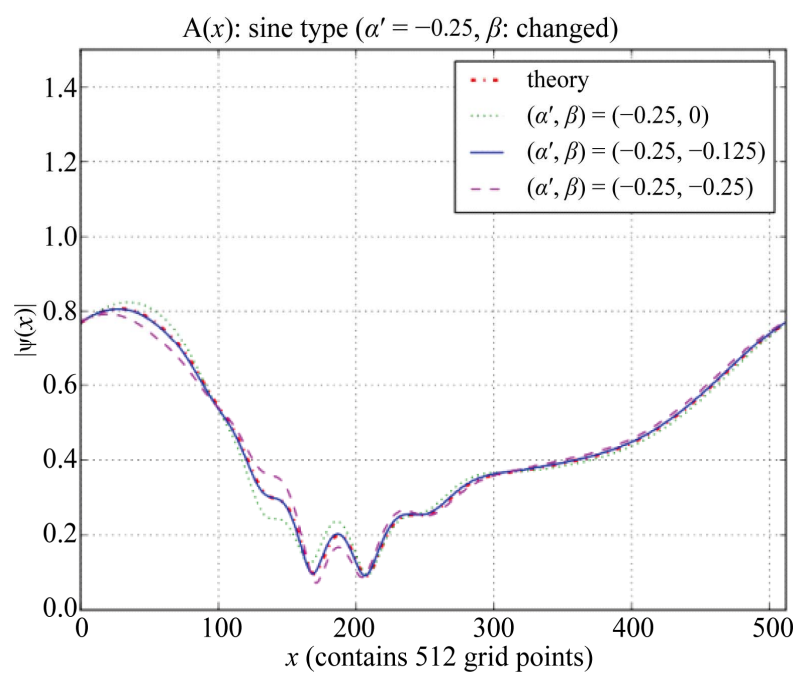

Figure 3. Parameter fitting for sine function type $A(x)$ (after 150000 walks) dash-dotted red: theoretical solution, dotted green: $\left(\alpha^{\prime}, \beta\right)=(-0.25,0)$, solid blue: $\left(\alpha^{\prime}, \beta\right)=(-0.25,-0.125)$, dashed magenta: $\left(\alpha^{\prime}, \beta\right)=(-0.25,-0.25)$. Here, $\alpha^{\prime}=-0.25$ is fixed and $\beta$ is varied. At $\beta=-0.125$, the quantum walk solution coincides with the theoretical solution. 512 grid points are used. Absolute values of two-point-averaged $\psi$ are plotted. $\left(\psi_{\text {ave } 2}(t, x)=(1 / 2)(\psi(t, x)+\psi(t, x+\Delta x))\right)$.

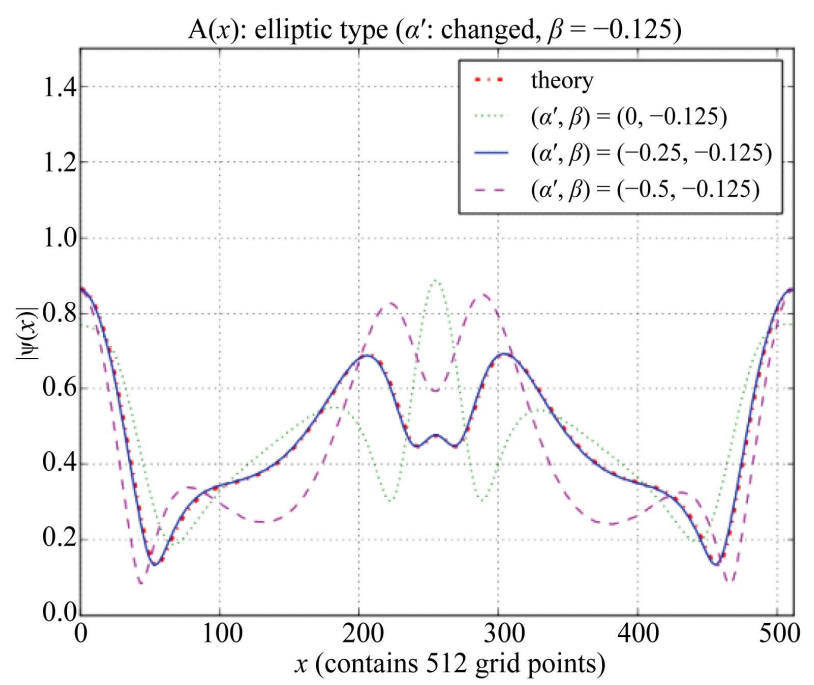

Figure 4. Parameter fitting for elliptic function type $A(x)$ (after 50000 walks) dash-dotted red: theoretical solution, dotted green: $\left(\alpha^{\prime}, \beta\right)=(0,-0.125)$, solid blue: $\left(\alpha^{\prime}, \beta\right)=(-0.25$, $-0.125)$, dashed magenta: $\left(\alpha^{\prime}, \beta\right)=(-0.5,-0.125)$. Here, $\beta=$ -0.125 is fixed and $\alpha^{\prime}$ is varied. At $\alpha^{\prime}=-0.25$, the quantum walk solution coincides with the theoretical solution. 512 grid points are used. Absolute values of two-point-averaged $\psi$ are plotted. $\left(\psi_{\text {ave } 2}(t, x)=(1 / 2)(\psi(t, x)+\psi(t, x+\Delta x))\right)$.

To summarize these results, we find that by selecting only two parameters $\alpha^{\prime}=-1 / 4, \beta=-1 / 8$, a theoretical solution and a quantum walk numerical solution coincides completely.

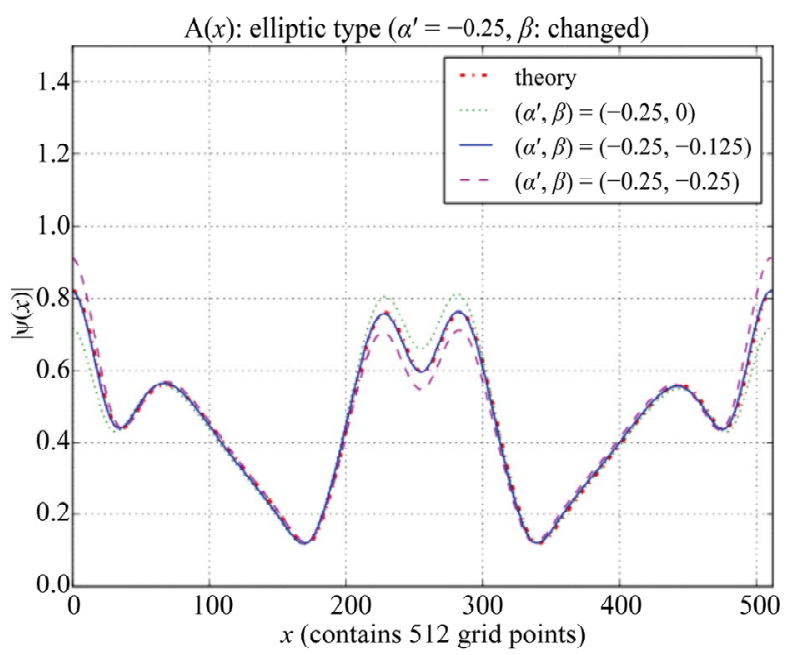

Figure 5. Parameter fitting for elliptic function type $A(x)$ (after 100000 walks) dash-dotted red: theoretical solution, dotted green: $\left(\alpha^{\prime}, \beta\right)=(-0.25,0)$, solid blue: $\left(\alpha^{\prime}, \beta\right)=(-0.25$, $-0.125)$, dashed magenta: $\left(\alpha^{\prime}, \beta\right)=(-0.25,-0.25)$. Here, $\alpha^{\prime}=$ -0.25 is fixed and $\beta$ is varied. At $\beta=-0.125$, the quantum walk solution coincides with the theoretical solution. 512 grid points are used. Absolute values of two-point-averaged $\psi$ are plotted. $\left(\psi_{\text {ave } 2}(t, x)=(1 / 2)(\psi(t, x)+\psi(t, x+\Delta x))\right)$.

Because $\alpha^{\prime}=\frac{1}{2}\left(\alpha-\frac{1}{2}\right), \beta=-\frac{1}{2}\left(\alpha-\frac{1}{2}\right)^{2}$ for $H_{\alpha}$, this result leads to $\alpha=0$.

Namely we find that $H_{0}=-\frac{1}{2} D A(x) A(x) D$ is the right Hamiltonian for the continuum limit evolution equation corresponding to the TDSE-type quantum walk with space dependent $\theta(x)$.

\section{Advection Equation with Space Dependent Velocity Field}

We consider space dependentparameters $(\theta(x))$ in the advection-type quantum walk (see Appendix A).

$$
U(x)=\left(\begin{array}{cc}
\cos \theta(x) & -\sin \theta(x) \\
\sin \theta(x) & \cos \theta(x)
\end{array}\right)
$$

Here we use $U(x)$ in place of $\mathrm{E}$ and $\mathrm{F}$ in Figure 1 and $\phi(x)=\frac{\pi}{2}, b(x)=0$ are chosen in Equation (1.5).

The evolution equation that emerges as the continuum limit for the advection-type quantum walk with space dependent velocity field $(A(x)=\sin \theta(x))$ is in general

$$
\begin{aligned}
& \frac{\partial \Psi}{\partial t}=A(x)^{1-\alpha} D A(x)^{\alpha} \Psi \\
& (=(\alpha D A(x)+(1-\alpha) A(x) D) \Psi) \\
& \left(\text { where } D \equiv \frac{\partial}{\partial x}\right)
\end{aligned}
$$


Especially $\alpha=0, \frac{1}{2}$ and 1 correspond to non-conserving-type, unitary-type and conserving-type advection equations respectively (Note that there is a relation $\psi=\sqrt{\rho}$ between unitary-type solution $\Psi$ and conserving-type solution $\rho$ ).

However since quantum walk is a unitary transformation, only the unitary-type advection equation is allowed.

In the following, we investigate using a numerical method if the unitary-type advection equation indeed emerges as a solution for continuum limit.

In our numerical method, we use periodic boundary condition for the range $[0,1]$, and use the following type of $A(x)$.

(More precisely, in the actual calculation the range $[0,1]$ was mapped to the range $[0,512])$

$$
A(x)=\frac{A_{0}}{1+\epsilon \sin (2 \pi x)}\left(A_{0}=0.5, \epsilon=0.5\right)
$$

and this satisfies

$$
\int_{0}^{1} \frac{\mathrm{d} x}{A(x)}=\frac{1}{A_{0}}
$$

In order to calculate the theoretical solution for

$$
\frac{\partial \psi(t, x)}{\partial t}=A(x)^{1-\alpha} D A(x)^{\alpha} \psi(t, x)
$$

we can simply reduce it to the solution of the constant velocity field $(A(x)=1)$ advection equation

$\psi_{0}(t, x)=F(t-x)$ (where $F(x)$ is an arbitrary periodic function of which period $=1)$.

$$
\begin{gathered}
\frac{\partial \psi_{0}(t, x)}{\partial t}=D \psi_{0}(t, x) \\
\psi(t, x)=\frac{\psi_{0}\left(t A_{0}, B(x)\right)}{A(x)^{\alpha}} \\
\text { where } B(x) \equiv \int_{0}^{x} \frac{A_{0}}{A\left(x^{\prime}\right)} \mathrm{d} x^{\prime}
\end{gathered}
$$

(Note that as $B(x+1)=B(x)+1$, the periodicity $\psi(t, x+1)=\psi(t, x)$ is guaranteed $)$.

We used $\psi_{0}(t=0, x)=\exp \left(-(10(x-0.5))^{2}\right)$ for the initial wave packet.

In fact, in the case of advection type quantum walk, both left moving and right moving components emerge. However we find that using the 4-point-averaging manipulation, we can extract only the one component (see Appendix C).

4-point-averaging is an averaging manipulation over four neighboring grid points in space-time.

$$
\begin{aligned}
& \psi_{\text {ave } 4}(t, x) \\
& \equiv \frac{1}{4}(\psi(t, x)+\psi(t, x+\Delta x) \\
& +\psi(t+\Delta t, x)+\psi(t+\Delta t, x+\Delta x))
\end{aligned}
$$

Now, if we assume $\psi(t, x) \approx \psi(t, x+\Delta x) \approx 1$ then

$$
\begin{aligned}
\left(\begin{array}{c}
\psi(t+\Delta t, x+\Delta x) \\
\psi(t+\Delta t, x)
\end{array}\right) & \approx\left(\begin{array}{cc}
\cos \theta & -\sin \theta \\
\sin \theta & \cos \theta
\end{array}\right)\left(\begin{array}{l}
1 \\
1
\end{array}\right) \\
& =\left(\begin{array}{l}
\cos \theta-\sin \theta \\
\cos \theta+\sin \theta
\end{array}\right)
\end{aligned}
$$

and therefore $\psi_{\text {ave } 4}(t, x) \approx \frac{1+\cos \theta}{2}=\cos ^{2} \frac{\theta}{2}$. It means that by 4-point-averaging the wave function scales with a factor of $\cos ^{2} \frac{\theta}{2}$.

In order to solve advection equations by using quantum walk with 4-point-averaging, we must consider this factor. It is not straightforward to deduce the right prescription to take into account the factor in a purely theoretical way. We here examined three different prescriptions to account the factor using numerical method.

Here we refer to the wave function of quantum walk as $\psi(t, x)$, and refer to the solution of the advection equation to be solved as $\tilde{\psi}(t, x)$.

Three methods we use are as follows (At initial time $(t=0), \tilde{\psi}(t=0, x)$ is loaded to $\psi(t=0, x)$ with/ without scaling factor, and at any time

$(t>0) \psi_{\text {ave } 4}(t, x)$ is copied to $\tilde{\psi}(t, x)$ with/without scaling factor for plotting).

$$
\begin{gathered}
\psi(t=0, x):=\frac{\tilde{\psi}(t=0, x)}{\cos ^{2} \frac{\theta}{2}}, \\
\tilde{\psi}(t, x):=\psi_{\text {ave } 4}(t, x) \\
\psi(t=0, x):=\frac{\tilde{\psi}(t=0, x)}{\cos \frac{\theta}{2}}, \\
\tilde{\psi}(t, x):=\frac{\psi_{\text {ave } 4}(t, x)}{\cos \frac{\theta}{2}} \\
\psi(t=0, x):=\tilde{\psi}(t=0, x), \\
\tilde{\psi}(t, x):=\frac{\psi_{\text {ave } 4}(t, x)}{\cos ^{2} \frac{\theta}{2}}
\end{gathered}
$$

(Method 1)

(Method 2)

(Method 3)

We show the numerical solutions in Figures 6-8 using the above prescriptions.

To summarize, from the numerical examination we find that method 2 is the right prescription. 


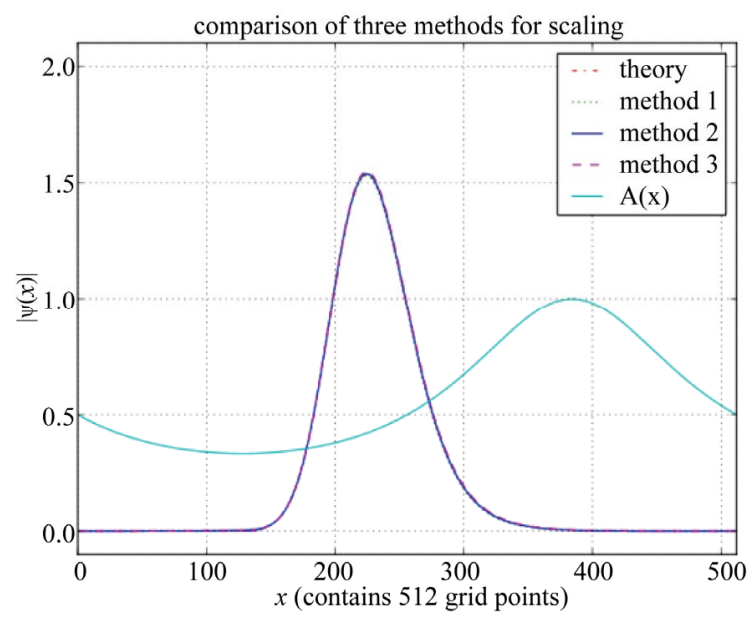

Figure 6. Comparison of three methods for different scalings (after 100 walks) dash-dotted red: theoretical solution, dotted green: method1 solid blue: method 2, dashed magenta: method 3, solid cyan: velocity $A(x)$. When the wave packet locates the region where $A(x)$ is small, the solution of all methods coincide with the theoretical solution with good accuracy.

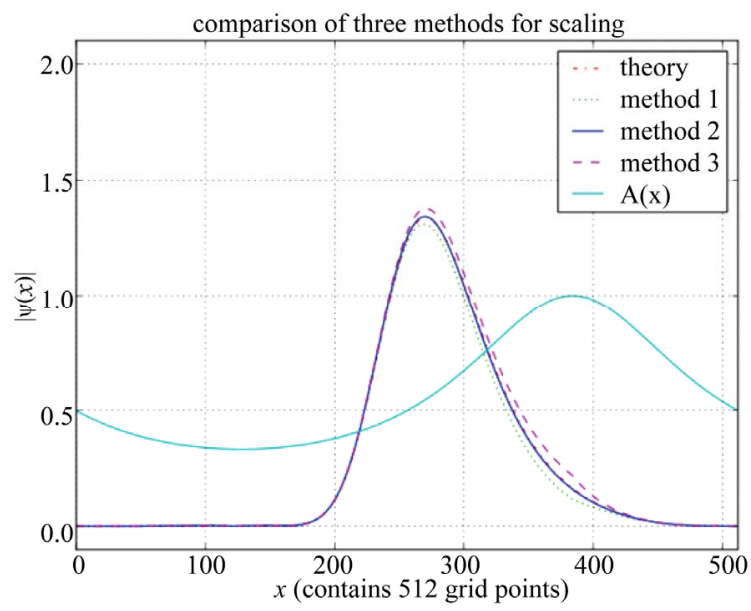

Figure 7. Comparison of three methods for different scalings (after 200 walks) dash-dotted red: theoretical solution, dotted green: method 1 solid blue: method 2, dashed magenta: method 3, solid cyan: velocity $A(x)$. As the wave packet comes close to the region where $A(x) \approx 1$, the differences among these methods become non-negligible and only the solution of method 2 coincides with the theoretical solution.

In general it is not so easy to solve an advection equation without numerical diffusion, but this method provides perfectly diffusion free solution by virtue of its unitarity.

\section{Conclusion}

The mathematics of quantum walk with variable parameters is not well established and difficult to derive space-time equation for its continuum limit by purely

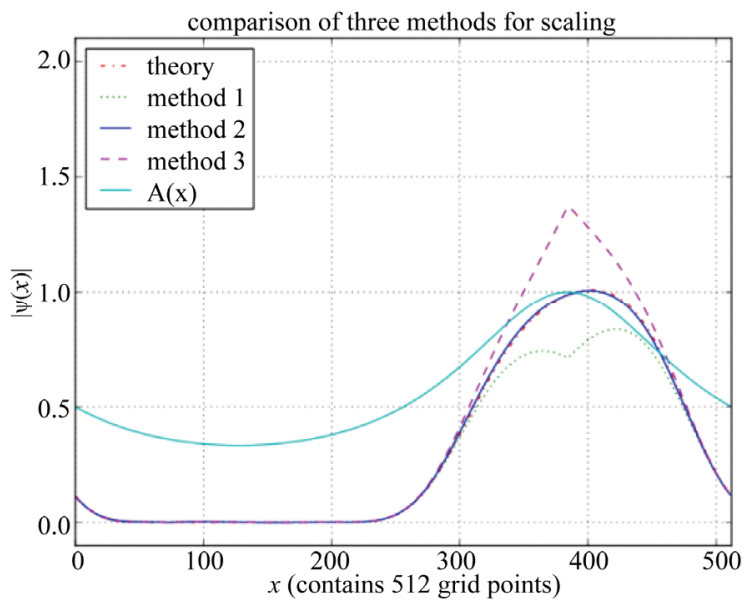

Figure 8. Comparison of three methods for different scalings (after 350 walks) dash-dotted red: theoretical solution, dotted green: method 1 solid blue: method 2, dashed magenta: method 3, solid cyan: velocity $A(x)$. When the wave packet locates around the region where $A(x) \approx 1$, the differences among these methods become large and only the solution of method 2 coincides with the theoretical solution.

mathematical method. And it is indispensable to compare theories with numerical methods especially in the case of space dependent parameters or broken translational invariance.

In this work, we propose clear-cut numerical methods to identify the right relation between the quantum walk with the space dependent parameters and the continuous space-time evolution equations. Using the method we establish the right relation between quantum walk and "TDSE with a space dependent imaginary diffusion coefficient" or "the advection equation with space dependent velocity fields".

Using the 4-point-averaging manipulation in the solution of advection equation by quantum walk, we find that only one component can be extracted out of two components of left-moving and right-moving solutions.

In the present work, we employ QCA formalism where extended space generated by combining original physical space and coin space (internal degree of freedom) is used.

On the extended discrete space, mathematics of quantum walks becomes more clear.

The extension to the multidimensional space is straightforward and currently we are applying the methodology to more realistic inhomogeneous quantum system in order to examine its practicality. Moreover our findings provide a clue to find more general space dependent formalisms such as solution method of TDSE with space dependent resolution by quantum walk/QCA.

\section{Acknowledgements}

This research was supported in part by TUT Programs on 
Advanced Simulation Engineering, Toyohashi University of Technology.

\section{REFERENCES}

[1] Y. Aharonov, L. Davidovich and N. Zagury, "Quantum Random Walks," Physical Review A, Vol. 48, No. 2, 1993, pp. 1687-1690. doi:10.1103/PhysRevA.48.1687

[2] N. Konno, "Mathematics of Quantum Walk," SangyoTosho, 2008.

[3] P. L. Knight, E. Roldán and J. E. Sipe, "Quantum Walk on the Line as Interference Phenomenon," Physical Review A, Vol. 68, No. 2, 2003, Article ID: 020301(R). doi:10.1103/PhysRevA.68.020301

[4] David A. Meyer, "Quantum Mechanics of Lattice gas Automation: One Particle Plane Waves and Potential," Physical Review E, Vol. 55, No. 5, 1997, pp. 5261-5269. doi:10.1103/PhysRevE.55.5261

[5] B. M. Boghosian and W. Taylor, "Quantum Lattice-Gas Model for the Many-Particle Schrödinger Equation in $\mathrm{d}$ Dimensions," Physical Review E, Vol. 57, No. 1, 1998, pp. 54-66. doi:10.1103/PhysRevE.57.54

[6] F. W. Strauch, "Relativistic Quantum Walks," Physical Review A, Vol. 73, No. 6, 2006, Article ID: 069908. doi:10.1103/PhysRevA.73.069908

[7] A. J. Bracken, D. Ellinas and I. Smyrnakis, "Free-Diracparticle Evolution as a Quantum Random Walk," Physical Review A, Vol. 75, No. 2, 2007, Article ID: 022322.

\section{doi:10.1103/PhysRevA.75.022322}

[8] C. M. Chandrashekar, S. Banerjee andR. Srikanth, "Relationship between Quantum Walks and Relativistic Quantum Mechanics," Physical Review A, Vol. 81, No. 6, 2010, Article ID: 062340. doi:10.1103/PhysRevA.81.062340

[9] A. Ahibrecht, H. Vogts, A. H. Werner, and R. F. Werner, "Asymptotic Evolution of Quantum Walks with Random Coin," Journal of Mathematical Physics, Vol. 52, No. 4, 2011, Article ID: 042201. doi:10.1063/1.3575568

[10] H. Sekino, M. Kawahata and S. Hamada, "A Solution of Time Dependent Schrödinger Equation by Quantum Walk," Journal of Physics Conference Series (JPCS), Vol. 352, No., 2012, Article ID: 012013. doi:10.1088/1742-6596/352/1/012013

[11] I. Daubechies, "Ten Lectures on Wavelets," SIAM, Philadelphia, 1992. doi:10.1137/1.9781611970104

[12] R. P. Feynman and A. R. Hibbs, "Quantum Mechanics and Path Integrals," McGraw Hill, 1965.

[13] R. Courant, K. Friedrichs and H. Lewy, "On the Partial Difference Equations of Mathematical Physics," IBM Journal of Research and Development, Vol. 11, No. 2, 1967, pp. 215-234.

[14] P. Høyer, "Efficient Quantum Transforms," Unpublished, 1997.

http://arxiv.org/abs/quant-ph/9702028v1 


\section{Appendix}

\section{Appendix A (Continuum Limit of Quantum Walk with Constant Parameters)}

Here we briefly review the way how an evolution equation can be derived as a continuum (zero wave number) limit of a quantum walk with constant parameters. This derivation technique is also used when 4-point-averaging is introduced (Appendix C).

In the method described here, the time evolution generator is expanded with respect to wavenumber $k$ around $k=0$. This treatment is essentially the same described in other literatures $[4,6]$.

In the latter, the effective mass

$m=\left[\frac{\mathrm{d}^{2} \omega(k)}{\mathrm{d} k^{2}}\right]_{k=0}^{-1}=\tan \theta$ was given from the dispersion relation $\cos \omega(k)=\cos \theta \cos k$ (though their model is different from ours and their $\theta$ corresponds to our $\frac{\pi}{2}-\theta$ ).

Note that the derivation in this appendix is not mathematically rigorous, we rather provide an outline of the derivation needed to explain or interpret the background and results of our numerical experiments.

We regard the wavefunction as a continuous function $\Psi(x)$, when the shape of the wavefunction varies slowly in space as compared with the grid spacing of the quantum walk. We show the continuous space-time evolution equation thus introduced.

First we consider the continuum limit of the classical random walk, classical counterpart of the quantum walk.

It is well known that by central limit theorem, the continuum limit of the classical random walk is a diffusion equation (If the left-right balance of the walk is broken, it leads to an advection-diffusion equation with an advective term)

$$
\frac{\partial \rho}{\partial t}=A \frac{\partial \rho}{\partial x}+B \frac{\partial^{2} \rho}{\partial x^{2}}
$$

First, we review how this equation can be derived.

We assume that the transition probability matrix $\hat{P}$ is translationally invariant. Namely $\hat{P}$ commutes with onegrid shift (to the negative direction) operation matrix $\hat{S}$.

Below is a simplest example of classical random walk with probabilities of both left and right walks being the equivalent value $1 / 2$.

Here we assume cyclic lattice of size $N$ or periodic boundary condition.

$$
\hat{P}=\left(\begin{array}{cccccc}
0 & 1 / 2 & 0 & 0 & \cdots & 1 / 2 \\
1 / 2 & 0 & 1 / 2 & 0 & \cdots & 0 \\
0 & 1 / 2 & 0 & 1 / 2 & \cdots & 0 \\
0 & 0 & 1 / 2 & 0 & \cdots & 0 \\
\vdots & \vdots & \vdots & \vdots & \ddots & 1 / 2 \\
1 / 2 & 0 & 0 & 0 & 1 / 2 & 0
\end{array}\right)
$$

$$
\hat{S}=\left(\begin{array}{cccccc}
0 & 1 & 0 & 0 & \cdots & 0 \\
0 & 0 & 1 & 0 & \cdots & 0 \\
0 & 0 & 0 & 1 & \cdots & 0 \\
0 & 0 & 0 & 0 & \cdots & 0 \\
\vdots & \vdots & \vdots & \vdots & \ddots & 1 \\
1 & 0 & 0 & 0 & 0 & 0
\end{array}\right)
$$

$$
[\hat{P}, \hat{S}]=0
$$

In treating translationally invariant discrete system, it is usual technique to diagonalize both $\hat{S}$ and $\hat{P}$ simultaneously using Z-transformation.

$$
P(s)=\sum_{i} \hat{P}_{0 i} s^{i}=\frac{1}{2}\left(s+s^{-1}\right)
$$

where $P(s)$ is the diagonal element of the diagonalized $\hat{P}$ corresponding to the eigenvalue s of $\hat{S}$.

Next we extend the problem from discrete time to continuous time and assume this leads to the following continuous time evolution equation

$$
\frac{\partial \rho(s)}{\partial t}=\bar{H}(s) \rho(s)
$$

(In this appendix we use $\bar{H}, H$ for generators $\frac{\partial}{\partial t}, i \frac{\partial}{\partial t}$ respectively).

As transition probability matrix for a unit time is $P(s)=e^{\bar{H}(s)}, \bar{H}(s)$ can be calculated by

$$
\bar{H}(s)=\log P(s)
$$

In order to investigate the continuum limit (or long range limit) behavior, we use the relation between the shift operator $(\hat{S})$ and the differential operator

$\left(D=\frac{\partial}{\partial x}\right) \hat{S}=e^{-D}$ and we have only to expand $H(s)$ in a Taylor series with respect to $\mathrm{k}$ of $s=e^{i k}$ around $k=0$. In this example,

$$
\begin{aligned}
\bar{H}(s) & =\log P(s)=\log (\cos k) \\
& =\log \left(1-\frac{k^{2}}{2}+o\left(k^{4}\right)\right)=-\frac{k^{2}}{2}+o\left(k^{4}\right)
\end{aligned}
$$

Therefore in the real space representation by replacing $-i k \rightarrow \frac{\partial}{\partial x}$ we obtain a diffusion equation

$$
\frac{\partial \rho}{\partial t}=\frac{1}{2} \frac{\partial^{2} \rho}{\partial x^{2}}
$$

In the case of quantum walk, we can use basically the same technique. the difference is that the quantum walk has not an 1-grid translational invariance but a 2-grid translational invariance. 


$$
\left[\hat{U}, \hat{S}^{2}\right]=0,[\hat{U}, \hat{S}] \neq 0
$$

We $2 \times 2$-block-diagonalize both $\hat{U}$ and $\hat{S}$ simultaneously by Z-transformation.

This time, unlike the case of classical random walk, we use Z-transformation of $2 \times 2$ matrix unit.

$$
\begin{aligned}
& U\left(s^{2}\right)=\sum_{i} \hat{U}_{0: 1,2 i: 2 i+1} s^{2 i} \\
& S\left(s^{2}\right)=\sum_{i} \hat{S}_{0: 1,2 i: 2 i+1} s^{2 i}=\left(\begin{array}{cc}
0 & 1 \\
s^{2} & 0
\end{array}\right)
\end{aligned}
$$

Here $\hat{U}_{0: 1,2 i: 2 i+1}$ denotes $2 \times 2$ submatrix ( 0 to 1 row indices and $2 i$ to $2 i+1$ column indices) of $\hat{U}$.

If we use the factorization form $\hat{U}=\hat{S}^{-1} \hat{E} \hat{S} \hat{F}$ of Figure 1.

Z-transformation of $U$ is obtained by the matrix multiplication of Z-transformation of each factor.

$$
\begin{aligned}
U\left(s^{2}\right) & =S\left(s^{2}\right)^{-1} E\left(s^{2}\right) S\left(s^{2}\right) F\left(s^{2}\right) \\
& =\left(\begin{array}{cc}
0 & s^{-2} \\
1 & 0
\end{array}\right) E\left(\begin{array}{cc}
0 & 1 \\
s^{2} & 0
\end{array}\right) F
\end{aligned}
$$

Now we assume the $2 \times 2$ matrix form of $E, F$ being

$$
\begin{aligned}
E=F & =\left(\begin{array}{ll}
A & B \\
C & D
\end{array}\right) \\
U\left(s^{2}\right) & =\left[\left(\begin{array}{cc}
0 & s^{-1} \\
s & 0
\end{array}\right)\left(\begin{array}{ll}
A & B \\
C & D
\end{array}\right)\right]^{2} \\
& =\left(\begin{array}{cc}
C s^{-1} & D s^{-1} \\
A s & B s
\end{array}\right)^{2}
\end{aligned}
$$

Then we regard the square root of $U\left(s^{2}\right)$ as 1-walk evolution matrix.

$$
U(s) \equiv \sqrt{U\left(s^{2}\right)}=\left(\begin{array}{cc}
C s^{-1} & D s^{-1} \\
A s & B s
\end{array}\right)
$$

The logarithm of $U(s)$ is obtained by decomposing $U(s)$ into the scalar part and the traceless part as follows.

$$
\begin{aligned}
& U(s) \equiv \alpha I+i \beta \Delta=\sqrt{\alpha^{2}+\beta^{2}} \exp (i \omega \Delta) \\
& \left(\omega \equiv \arctan \left(\frac{\beta}{\alpha}\right)=\arccos \left(\frac{\alpha}{\sqrt{\alpha^{2}+\beta^{2}}}\right)\right)
\end{aligned}
$$

$(\alpha, \beta, \omega \in C, \Delta: 2 \times 2$ matrix with trace $(\Delta)=0$ and $\left.\Delta^{2}=I\right)$

$$
\begin{aligned}
\bar{H}(s) & \equiv \log U(s)=\log (\alpha I+i \beta \Delta) \\
& =\log \sqrt{\alpha^{2}+\beta^{2}}+i \Delta \omega
\end{aligned}
$$

As $\Delta$ has eigenvalues +1 and -1 , we can obtain $\alpha, \beta$ from

$$
\begin{aligned}
& \operatorname{trace}(U(s))=C s^{-1}+B s=2 \alpha \\
& \operatorname{det}(U(s))=-(A D-B C) \\
& =(\alpha+i \beta)(\alpha-i \beta)=\alpha^{2}+\beta^{2}
\end{aligned}
$$

Now we consider more concrete form for $(A, B, C, D)$.

$$
\begin{aligned}
\left(\begin{array}{ll}
A & B \\
C & D
\end{array}\right) & =\exp \left(i \theta\left(\sigma_{x} \cos \phi+\sigma_{y} \sin \phi\right)-i b\right) \\
& =\left(\begin{array}{cc}
\cos \theta & i \sin \theta e^{-i \phi} \\
i \sin \theta e^{i \phi} & \cos \theta
\end{array}\right) e^{-i b}
\end{aligned}
$$

Here, $\sigma_{x}=\left(\begin{array}{ll}0 & 1 \\ 1 & 0\end{array}\right), \sigma_{y}=\left(\begin{array}{cc}0 & -i \\ i & 0\end{array}\right)$ are Pauli's matrices.

Then using $s=e^{i k}$ we have

$$
\begin{gathered}
\alpha=\frac{1}{2} \operatorname{trace}(U(s))=i \sin \theta \cos (\phi-k) e^{-i b} \\
\sqrt{\alpha^{2}+\beta^{2}}=\sqrt{\operatorname{det}(U(s))}=\mathrm{i} e^{-i b}
\end{gathered}
$$

and therefore

$$
\begin{aligned}
\bar{H}(s) & =\left(\frac{\pi}{2}-b\right) i+i \Delta \omega, \\
\omega=\arccos (\sin \theta \cos (\phi-k))(0<\omega<\pi) & \\
\sin \omega & \equiv \sqrt{1-\sin ^{2} \theta \cos ^{2}(\phi-k)} \\
& =\sqrt{\cos ^{2} \theta+\sin ^{2} \theta \sin ^{2}(\phi-k)} \\
-\Delta & \equiv \frac{1}{\sin \omega}\left(\begin{array}{cc}
-\sin \theta \sin (\phi-k) & \cos \theta e^{i k} \\
\cos \theta e^{-i k} & \sin \theta \sin (\phi-k)
\end{array}\right)
\end{aligned}
$$

In order to investigate the continuum limit (or long range limit) behavior, we expand $\omega(s)$ in a Taylor series with respect to $k$ of $s=e^{i k}$ around $k=0$ and we have

$$
\begin{aligned}
& \omega\left(e^{i k}\right) \\
& =\arccos (\sin \theta \cos (\phi-k)) \\
& =\arccos (\sin \theta \cos \phi)-\frac{\sin \theta \sin \phi}{\sqrt{1-\sin ^{2} \theta \cos ^{2} \phi}} k \\
& +\frac{1}{2} \frac{\sin \theta \cos ^{2} \theta \cos \theta}{\left(1-\sin ^{2} \theta \cos ^{2} \phi\right)^{\frac{3}{2}}} k^{2}+o\left(k^{3}\right)
\end{aligned}
$$

Here we use the Taylor expansion of arccos,

$$
\begin{aligned}
& \arccos (a+x) \\
& =\arccos (a)-\frac{x}{\sqrt{1-a^{2}}}-\frac{1}{2} \frac{a x^{2}}{\left(1-a^{2}\right)^{\frac{3}{2}}}+o\left(x^{3}\right) \\
& (0<\arccos <\pi) .
\end{aligned}
$$


Two cases ( $\phi=0$ or $\left.\phi=\frac{\pi}{2}\right)$ are particularly important, and from now on we restrict our arguments to these cases.

$$
\begin{aligned}
& \omega\left(e^{i k}\right)=\arccos (\sin \theta \cos k) \\
& =\frac{\pi}{2}-\theta+\frac{1}{2} k^{2} \tan \theta+o\left(k^{4}\right) \\
& \text { ( for } \phi=0 \text { ) } \\
& \omega\left(e^{i k}\right)=\arccos (\sin \theta \sin k) \\
& =\frac{\pi}{2}-k \sin \theta+o\left(k^{3}\right) \\
& \left(\text { for } \phi=\frac{\pi}{2}\right) \\
& -\Delta(k=0)=\left(\begin{array}{ll}
0 & 1 \\
1 & 0
\end{array}\right),\left(\begin{array}{cc}
-\sin \theta & \cos \theta \\
\cos \theta & \sin \theta
\end{array}\right) \\
& \text { (for } \phi=0, \frac{\pi}{2} \text { respectively) }
\end{aligned}
$$

Now we consider eigenfunctions of $\Delta(k)$ or (which is the same) $\bar{H}\left(e^{i k}\right)$

$$
-\Delta\left(e^{i k}\right) \psi^{( \pm)}(k)= \pm \psi^{( \pm)}(k)
$$

The equation of motion for wavenumber representation $\psi^{( \pm)}(t, k)$ is

$$
\begin{aligned}
\frac{\partial \psi^{( \pm)}}{\partial t} & =\bar{H} \psi^{( \pm)} \\
& \approx-i\left(\left(b-\frac{\pi}{2}\right) \pm\left(\frac{\pi}{2}-\theta+\frac{1}{2} k^{2} \tan \theta\right)\right) \Psi^{( \pm)}
\end{aligned}
$$

(for $\phi=0$ )

$$
\begin{aligned}
& \frac{\partial \psi^{( \pm)}}{\partial t}=\bar{H} \psi^{( \pm)} \approx\left(i\left(\frac{\pi}{2} \mp \frac{\pi}{2}\right) \mp \sin \theta \frac{\partial}{\partial x}\right) \Psi^{( \pm)} \\
& \left(\text {for } \phi=\frac{\pi}{2} \text { and } b=0\right)
\end{aligned}
$$

So the equation of motion for real space representation $\psi^{( \pm)}(t, x)$ is

$$
\begin{aligned}
& i \frac{\partial \psi^{( \pm)}}{\partial t}=H \psi^{( \pm)} \\
& \approx\left(\left(b-\frac{\pi}{2}\right) \pm\left(\frac{\pi}{2}-\theta-\frac{1}{2} \tan \theta \frac{\partial^{2}}{\partial x^{2}}\right)\right) \Psi^{( \pm)} \\
& (\text {or } \phi=0) \\
& \frac{\partial \psi^{( \pm)}}{\partial t}=\bar{H} \psi^{( \pm)} \approx\left(i\left(\frac{\pi}{2} \mp \frac{\pi}{2}\right) \mp \sin \theta \frac{\partial}{\partial x}\right) \Psi^{( \pm)} \\
& \left(\text {for } \phi=\frac{\pi}{2} \text { and } b=0\right)
\end{aligned}
$$

Thus we obtain the TDSE with potential term ( for $\phi=0$ ) and advection equation.

$$
\left(\text { for } \phi=\frac{\pi}{2} \text { and } b=0\right) \text {. }
$$

Here we drop higher-order terms.

Now we describe the physical meaning of $\psi^{( \pm)}$ briefly.

For TDSE-type $(\phi=0),-\Delta(k=0)=\left(\begin{array}{ll}0 & 1 \\ 1 & 0\end{array}\right)$ and its eigenvector are $\left(\begin{array}{c}1 \\ \pm 1\end{array}\right)$.

It is plausible that the eigenvector $\left(\begin{array}{l}1 \\ 1\end{array}\right)$ corresponds to the wave function slowly varying in space, namely $\psi(t, x) \approx \psi(t, x+\Delta x)$.

On the contrary, for advection-type

$\left(\phi=\frac{\pi}{2}\right),-\Delta(k=0)=\left(\begin{array}{cc}-\sin \theta & \cos \theta \\ \cos \theta & \sin \theta\end{array}\right)$ and $\left(\begin{array}{l}1 \\ 1\end{array}\right)$ is not the eigenvector, so the wave function slowly varying in space must have both $\Psi^{(+)}$and $\Psi^{(-)}$components corresponding to left-moving and right-moving wave packets.

In Figure A1 we plot the dispersion relation for the TDSE type quantum walk (Equation (A.25)).

Note that if we shift the horizontal axis by $-\frac{\pi}{2}$, we can regard this graph as the dispersion relation for advection type quantum walk (Equation (A. 26)).

In the TDSE case, as $\theta$ comes close to $\frac{\pi}{2}$, the dispersion relation around $k \approx 0$ changesfrom quadratic to linear and this corresponds to the one-dimensional Dirac equation with a small mass $m=\frac{1}{\tan \theta} \approx \frac{\pi}{2}-\theta$.

This can be seen as follows (shown by the article [6]).

$$
\begin{aligned}
& \bar{H}=\log \left(\left(\begin{array}{cc}
0 & s^{-1} \\
s & 0
\end{array}\right) \exp \left(i \theta \sigma_{x}\right)\right) \\
&=\log \left(\exp \left(-i k \sigma_{z}\right) \sigma_{x} \exp \left(i \theta \sigma_{x}\right)\right) \\
& \approx \log \left(i \exp \left(-i k \sigma_{z}-i\left(\frac{\pi}{2}-\theta\right) \sigma_{x}\right) ?\right) \\
&=-i\left(-\frac{\pi}{2}+k \sigma_{z}+\left(\frac{\pi}{2}-\theta\right) \sigma_{x}\right) \\
&\left(\text { if both }\left(\frac{\pi}{2}-\theta\right) \text { and } k \text { are small }\right)
\end{aligned}
$$

Therefore in a real space representation without the constant phase rotation factor $-\frac{\pi}{2}$ in Equation (A.33), 


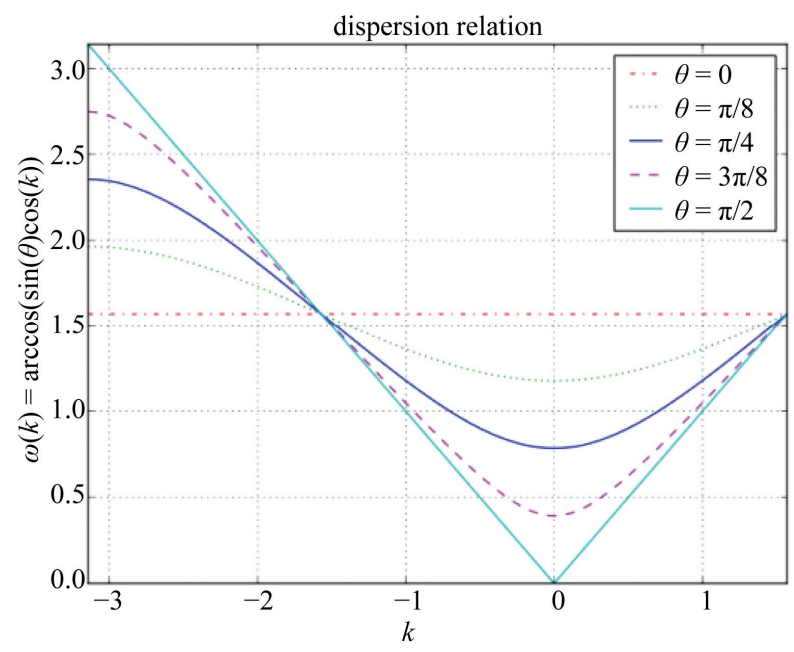

Figure A1. dispersion relation for TDSE (or advection) type quantum walk.

we have

$$
i \frac{\partial \psi}{\partial t}=\left[-i \sigma_{z} \frac{\partial}{\partial x}+\left(\frac{\pi}{2}-\theta\right) \sigma_{x}\right] \psi
$$

Finally we comment about unit system. By comparing the continuum limit evolution equation for the TDSEtype quantum walk with $\theta$ and $b$

$$
\begin{aligned}
& i \frac{\partial \psi^{(+)}}{\partial t} \\
= & H \psi^{(+)} \\
= & \left(-\frac{1}{2} \tan \theta \frac{\partial^{2}}{\partial x^{2}}+b-\theta\right) \psi^{(+)}
\end{aligned}
$$

and the usual TDSE

$$
i \frac{\partial \psi}{\partial t}=\mathrm{H} \psi=\left(-\frac{1}{2 m} \frac{\partial^{2}}{\partial x^{2}}+V\right) \psi
$$

we can say that the correspondence relation $\frac{1}{m} \approx \tan \theta$ and $V \approx b-\theta$ hold. It is true if we use the units system of $\Delta t=\Delta x=1$ where $\Delta t$ means the time interval of each step and $\Delta x$ means the grid interval. If we use more general units system, we must say that $\frac{1}{m} \frac{\Delta t}{(\Delta x)^{2}} \approx \tan \theta$ and $V \Delta t \approx b-\theta$ using dimensionless quantities.

Throughout this article we call $\frac{1}{2 m}$ as imaginary diffusion coefficient.

One more important comment to say is that in a finite difference (leap-frog) method

$$
\begin{aligned}
& i \frac{\psi(t+\Delta t, x)-\psi(t-\Delta t, x)}{2 \Delta t} \\
& =\frac{1}{2 m} \frac{\psi(t, x+\Delta x)+\psi(t, x-\Delta x)-2 \psi(t, x)}{(\Delta x)^{2}}
\end{aligned}
$$

there is a sharp stability condition (CFL condition) for nondimensionalized imaginary diffusion coefficient

$\frac{1}{m} \frac{\Delta t}{(\Delta x)^{2}} \leq \frac{1}{2}[13]$, but in quantum walk there is no such limitation.

\section{Appendix B (Factorization of Unitary Banded Matrix)}

Here we briefly review the factorization of 2-grid translationally invariant unitary banded matrix.

Factorization of matrix can be found in articles such as [14].

We will show

$$
\hat{S}^{-2}\left(\hat{A}+\hat{B} \hat{S}^{2}+\hat{C} \hat{S}^{4}\right)=\left(\hat{S}^{-2} \hat{D} \hat{S}^{2}\right)\left(\hat{S}^{-1} \hat{E} \hat{S}\right) \hat{F}
$$

that corresponds to Figure A2, or equivalently.

$$
\hat{A}+\hat{B} \hat{S}^{2}+\hat{C} \hat{S}^{4}=\hat{D} \hat{S} \hat{E} \hat{S} \hat{F}
$$

(Note that $\hat{S}^{2}$ commutes with any matrix dealt here). By the unitarity

$$
\left(\hat{A}+\hat{B} \hat{S}^{2}+\hat{C} \hat{S}^{4}\right)^{+}\left(\hat{A}+\hat{B} \hat{S}^{2}+\hat{C} \hat{S}^{4}\right)=\hat{I}
$$

or equivalently (after Z-transformation)

$$
\begin{aligned}
& \left(\mathrm{A}+\mathrm{Bs}^{2}+C s^{4}\right)^{+}\left(\mathrm{A}+\mathrm{Bs}^{2}+C s^{4}\right) \\
& =\left(\mathrm{A}^{+} \mathrm{A}+\mathrm{B}^{+} \mathrm{B}+\mathrm{C}^{+} \mathrm{C}\right)+\left(\mathrm{A}^{+} \mathrm{B}+\mathrm{B}^{+} \mathrm{C}\right) s^{2} \\
& \quad+\left(\mathrm{B}^{+} \mathrm{A}+\mathrm{C}^{+} \mathrm{B}\right) s^{-2}+\mathrm{A}^{+} \mathrm{C} s^{4}+\mathrm{C}^{+} \mathrm{As}^{-4}=\mathrm{I}
\end{aligned}
$$

we can get

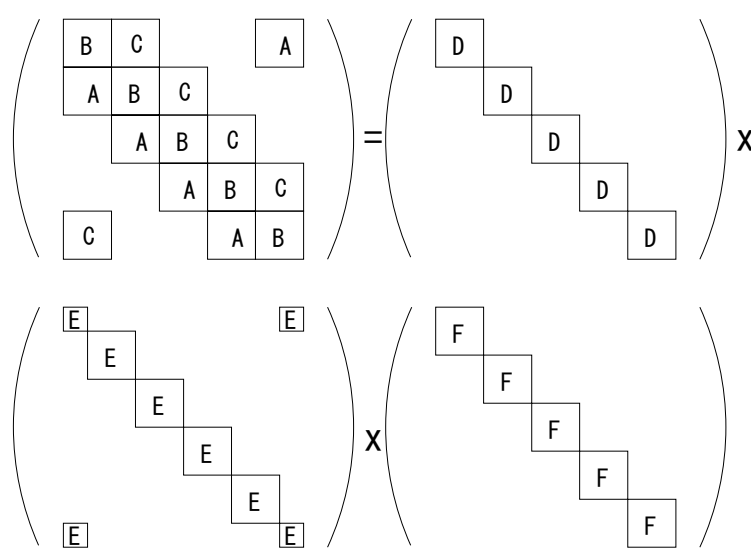

Figure A2. Factorization of unitary banded matrix. 


$$
\begin{aligned}
& A^{+} A+B^{+} B+C^{+} C=I \\
& A^{+} B+B^{+} C=B^{+} A+C^{+} B=0 \\
& C^{+} A=A^{+} C=0
\end{aligned}
$$

(Here superscript $(+)$ denotes Hermitian conjugate).

Now we define Hermitian matrices $P=A A^{+}, Q=C C^{+}$.

As $P Q=Q P=0$ and $P, Q$ can be diagonalized simultaneously by some unitary matrix $D^{+}$, diagonalized $P$, $Q$ have the forms of

$$
D^{+} P D=\left(\begin{array}{cc}
\lambda_{1} & 0 \\
0 & 0
\end{array}\right), D^{+} Q D=\left(\begin{array}{ll}
0 & 0 \\
0 & \lambda_{2}
\end{array}\right)
$$

Namely

$$
\begin{aligned}
& \left(D^{+} A\right)\left(D^{+} A\right)^{+}=\left(\begin{array}{ll}
\lambda_{1} & 0 \\
0 & 0
\end{array}\right) \\
& \left(D^{+} C\right)\left(D^{+} C\right)^{+}=\left(\begin{array}{ll}
0 & 0 \\
0 & \lambda_{2}
\end{array}\right)
\end{aligned}
$$

Therefore, $D^{+} A$ and $D^{+} C$ should have the form of

$$
\begin{gathered}
D^{+} A=\left(\begin{array}{ll}
a & b \\
0 & 0
\end{array}\right), D^{+} C=\left(\begin{array}{ll}
0 & 0 \\
c & d
\end{array}\right) \\
\left(|a|^{2}+|b|^{2}=\lambda_{1},|c|^{2}+|d|^{2}=\lambda_{2}\right)
\end{gathered}
$$

the above equations can be illustrated as Figure A3. (both lower half of $A$ and upper half of $C$ are zero).

Namely by applying from the left $D^{+}$to $U$, the band width can be reduced from 3-block-width to 2-blockwidth.

This can be expressed by

$$
D^{+}\left(\hat{A}+\hat{B} \hat{S}^{2}+\hat{C} \hat{S}^{4}\right)=\hat{S}\left(\hat{X}+\hat{Y} \hat{S}^{2}\right)
$$

or

$$
\hat{A}+\hat{B} \hat{S}^{2}+\hat{C} \hat{S}^{4}=\hat{D} \hat{S}\left(\hat{X}+\hat{Y} \hat{S}^{2}\right)
$$

Next similar procedure can be applied to $\left(\hat{X}+\hat{Y} \hat{S}^{2}\right)$ and we can say that

$$
\hat{X}+\hat{Y} \hat{S}^{2}=\hat{E} \hat{S} \hat{F}
$$

so finally Equation (B.2)

$$
\hat{A}+\hat{B} \hat{S}^{2}+\hat{C} \hat{S}^{4}=\hat{D} \hat{S} \hat{E} \hat{S} \hat{F}
$$

is obtained.

\section{Appendix C (4-Point-Averaging)}

4-point-averaging is an averaging manipulation over four neighboring grid points in space-time.

$$
\begin{aligned}
& \psi_{\mathrm{ave} 4}(t, x) \\
& \equiv \frac{1}{4}(\psi(t, x)+\psi(t, x+\Delta x) \\
& +\psi(t+\Delta t, x)+\psi(t+\Delta t, x+\Delta x))
\end{aligned}
$$

Now we let the $2 \times 2$ unitary matrix of a constant parameter quantum walk $U=\left(\begin{array}{cc}A & B \\ C & D\end{array}\right)$ and let $o, p, q, r, s$, $t, u, v, w, x, y, z$ be the wave function values at grid points as shown in Figure A4.

Namely

$$
\begin{aligned}
& \left(\begin{array}{l}
r \\
s
\end{array}\right)=\left(\begin{array}{ll}
A & B \\
C & D
\end{array}\right)\left(\begin{array}{l}
o \\
p
\end{array}\right),\left(\begin{array}{l}
u \\
v
\end{array}\right)=\left(\begin{array}{ll}
A & B \\
C & D
\end{array}\right)\left(\begin{array}{l}
q \\
r
\end{array}\right), \\
& \left(\begin{array}{l}
w \\
x
\end{array}\right)=\left(\begin{array}{ll}
A & B \\
C & D
\end{array}\right)\left(\begin{array}{l}
s \\
t
\end{array}\right),\left(\begin{array}{l}
y \\
z
\end{array}\right)=\left(\begin{array}{ll}
A & B \\
C & D
\end{array}\right)\left(\begin{array}{l}
v \\
w
\end{array}\right)
\end{aligned}
$$

and let $a, c, d, e$ be their 4-point-averaged values.

$$
\begin{aligned}
& a=(q+r+u+v) / 4 \\
& c=(s+t+w+x) / 4 \\
& d=(o+p+r+s) / 4 \\
& e=(v+w+y+z) / 4
\end{aligned}
$$

$a, c, d, e$, can be represented by $q, r, s, t$ as follows.

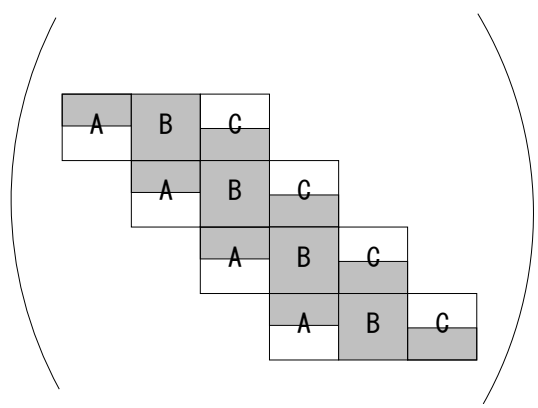

Figure A3. Band-width reduction of unitary banded matrix.

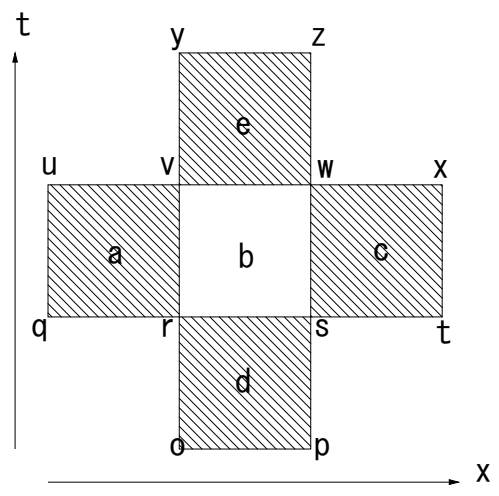

Figure A4. Illustration of 4-point averaging. 


$$
\left(\begin{array}{l}
a \\
c \\
e \\
d
\end{array}\right)=\left(\begin{array}{cccc}
1+A+C & 1+B+D & 0 & 0 \\
0 & 0 & 1+A+C & 1+B+D \\
C(1+A+C) & D(1+A+C) & A(1+B+D) & B(1+B+D) \\
0 & 1+(D-C) / \delta & 1+(\mathrm{A}-B) / \delta & 0
\end{array}\right)\left(\begin{array}{l}
q \\
r \\
s \\
t
\end{array}\right)
$$

where $\delta=A D-B C$

By a simple calculation, we can find that this matrix is singular and can have a relation

$$
e-C a-B c=\delta d
$$

If we regard the change $(a, d, c) \rightarrow(a, e, c)$ as a onestep time evolution, overall one-step evolution equation can be written as follows (We refer to this matrix as $\hat{F}$ ).

$$
\left(\begin{array}{l}
a \\
e \\
c \\
* \\
* \\
*
\end{array}\right)=\left(\begin{array}{ccccccc}
1 & & & & & \\
C & \delta & B & & & \\
& & 1 & & & \\
& & C & \delta & B & \\
& & & & 1 & \\
& & & & C & \delta
\end{array}\right)\left(\begin{array}{c}
a \\
d \\
c \\
* \\
* \\
*
\end{array}\right)
$$

Using shift matrix $\hat{S}$, two-step evolution matrix can be written as

$$
\hat{T}=\hat{S}^{-1} \hat{F} \hat{S} \hat{F}
$$

And Z-transformation (by $2 \times 2$ matrix unit) of this is obtained as

$$
\begin{aligned}
& T\left(s^{2}\right)=S\left(s^{2}\right)^{-1} F\left(s^{2}\right) S\left(s^{2}\right) F\left(s^{2}\right) \\
& =\left(\begin{array}{cc}
0 & s^{-2} \\
1 & 0
\end{array}\right)\left(\begin{array}{cc}
1 & 0 \\
C+B s^{2} & \delta
\end{array}\right)\left(\begin{array}{cc}
0 & 1 \\
s^{2} & 0
\end{array}\right)\left(\begin{array}{cc}
1 & 0 \\
C+B s^{2} & \delta
\end{array}\right) \\
& =\left(\begin{array}{cc}
C s^{-1}+D s & \delta s^{-1} \\
s & 0
\end{array}\right)
\end{aligned}
$$

Now we consider its square root as 1-step evolution matrix.

$$
T(s) \equiv \sqrt{T\left(s^{2}\right)}=\left(\begin{array}{cc}
C s^{-1}+B s & \delta s^{-1} \\
s & 0
\end{array}\right)
$$

Comparing with the Z-transformation of the original quantum walk

$$
U(s)=\left(\begin{array}{cc}
C s^{-1} & D s^{-1} \\
A s & B s
\end{array}\right)
$$

we can find that

$$
\begin{aligned}
& \operatorname{det}(T(s))=\operatorname{det}(U(s))=-\delta, \\
& \operatorname{trace}(T(s))=\operatorname{trace}(U(s))=C s^{-1}+B s
\end{aligned}
$$

and therefore both $T(s)$ and $U(s)$ have the same eigenvalues.

Therefore if we use

$$
\begin{aligned}
\left(\begin{array}{ll}
A & B \\
C & D
\end{array}\right) & =\exp \left(i \theta\left(\sigma_{x} \cos \phi+\sigma_{y} \sin \phi\right)-i b\right) \\
& =\left(\begin{array}{cc}
\cos \theta & i \sin \theta e^{-i \phi} \\
i \sin \theta e^{i \phi} & \cos \theta
\end{array}\right) e^{-i b}
\end{aligned}
$$

we can derive TDSE or advection equation for $\phi=0$ and $\phi=\frac{\pi}{2}$ respectively in the same way shown in Appendix A.

The only difference between $T(s)$ and $U(s)$ is the eigenfunctions of $\Delta\left(e^{i k}\right)$.

In this case $(T(s))$

$$
\begin{aligned}
& \Delta(k=0)=\frac{1}{\cos \theta}\left(\begin{array}{cc}
i \sin \theta & 1 \\
1 & -i \sin \theta
\end{array}\right),\left(\begin{array}{ll}
0 & 1 \\
1 & 0
\end{array}\right) \\
& \left(\text { for } \phi=0, \frac{\pi}{2} \text { respectively }\right)
\end{aligned}
$$

Therefore this time, the advection-type quantum walk has an eigenvector $\left(\begin{array}{l}1 \\ 1\end{array}\right)$ and we can assume that the wave function spatially-varying slowly must have only one component out of two $\left(\Psi^{( \pm)}\right)$components. 